Supporting Information

\title{
2D $\mathrm{Ln}^{\mathrm{III}} \mathrm{Ru}^{\mathrm{III}}{ }_{2}$ Compounds Constructed from trans-[Ru(acac $\left.)_{2}(\mathrm{CN})_{2}\right]^{-}$. Syntheses, Structures and Magnetic Properties
}

Wai-Fun Yeung,,$^{\dagger}$ Tai-Chu Lau, ${ }^{* \dagger}$ Xin-Yi Wang ${ }^{+}$, Song Gao, ${ }^{*+\dot{t}}$ Lap Szeto ${ }^{\S}$ and Wing-Tak Wong ${ }^{\S}$

Department of Biology and Chemistry, City University of Hong Kong, Tat Chee Avenue, Kowloon Tong, Hong Kong, China, Beijing National Laboratory for Molecular Sciences, State Key Laboratory of Rare Earth Materials Chemistry and Applications, College of Chemistry and Molecular Engineering, Peking University, Beijing 100871, China, and Department of Chemistry, the University of Hong Kong, Pokfulam Rd., Hong Kong, China 


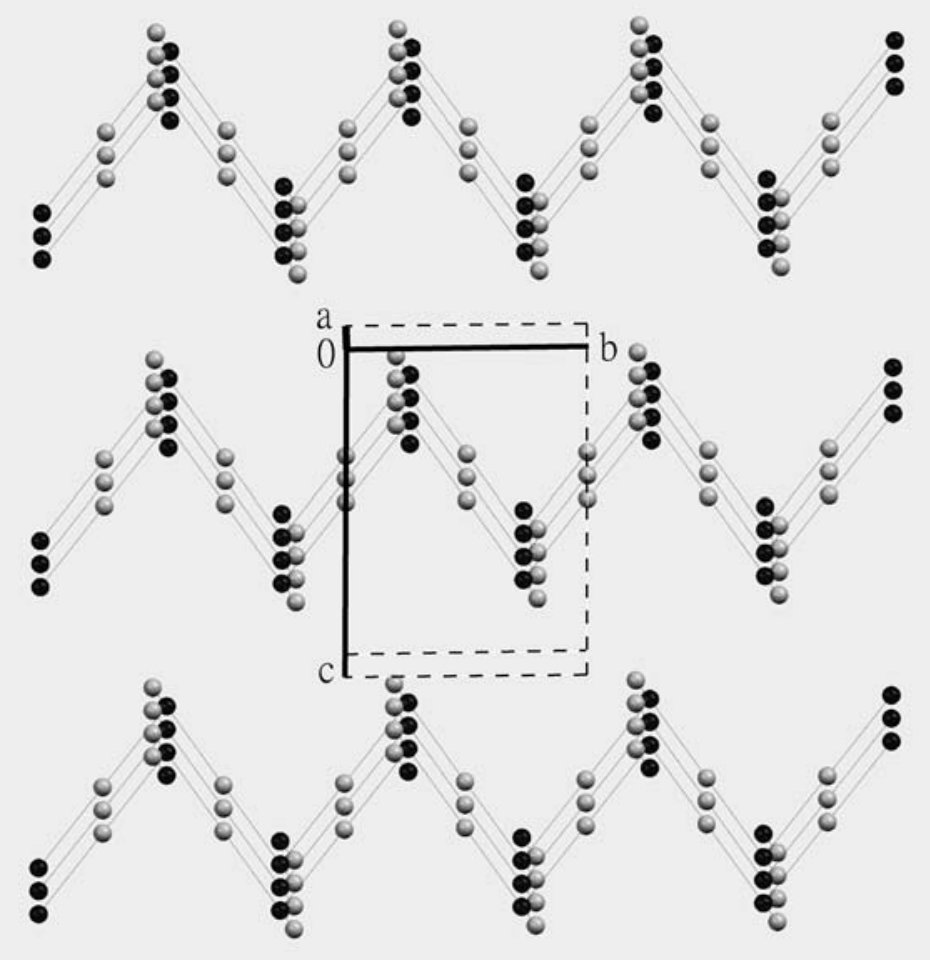

Figure S1. Arrangement of $\mathrm{Tb}(\mathrm{III})$ and $\mathrm{Ru}(\mathrm{III})$ atoms of adjacent planes viewed along $a$-axis showing the face-to-face stacking (other atoms are omitted for clarity). 


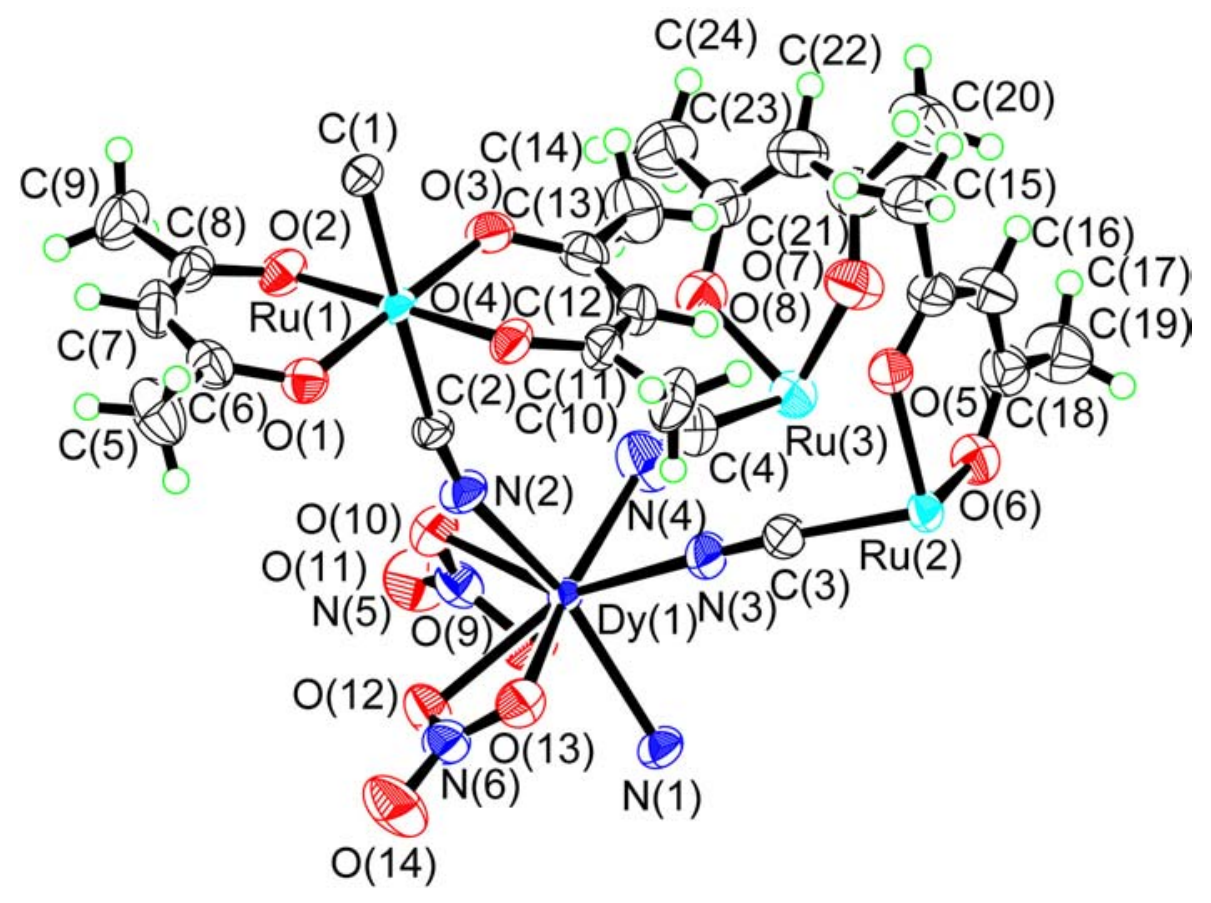

Figure S2. The ORTEP diagram of 2. 


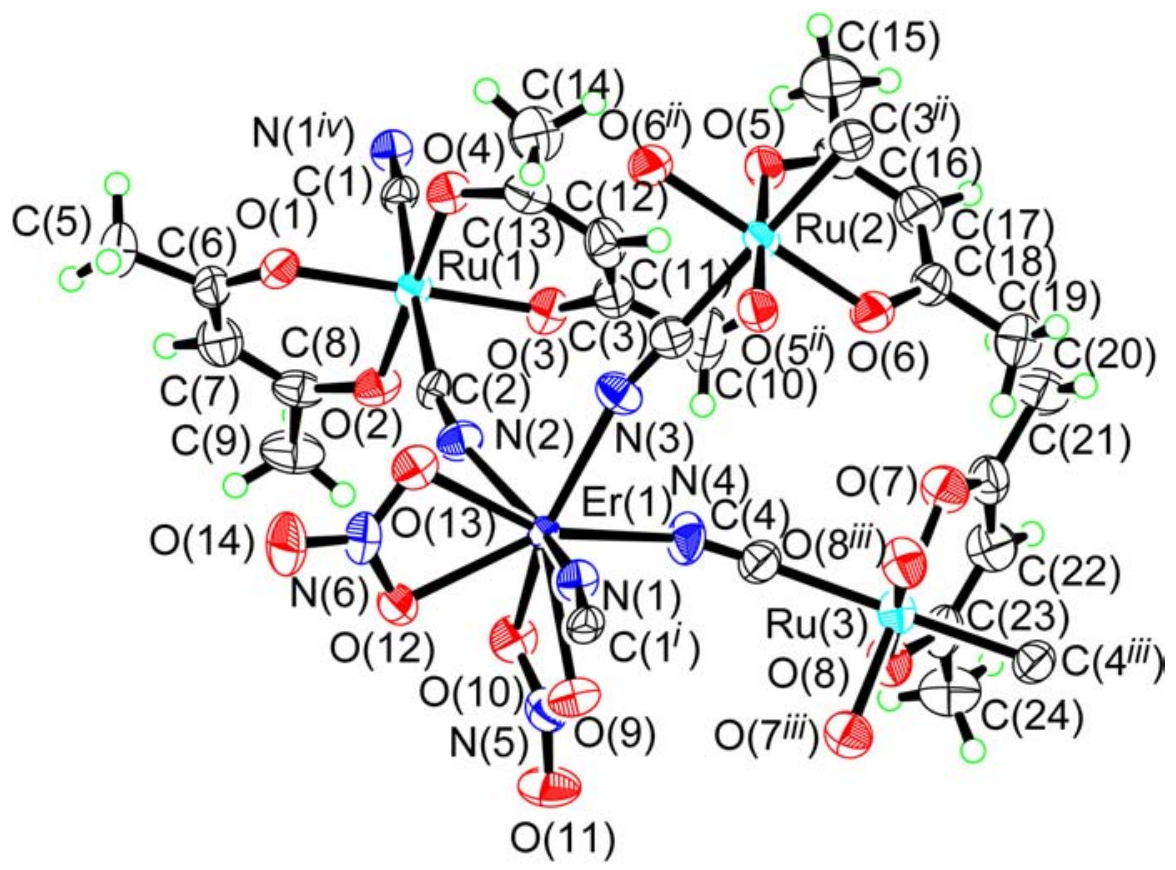

Symmetry code: (i) $1+x, y, z$; (ii) 1-x, -y, 1-z; (iii) 1-x, 1-y, 1-z (iv) $x-1, y, z$.

Figure S3. The ORTEP diagram of 3. 


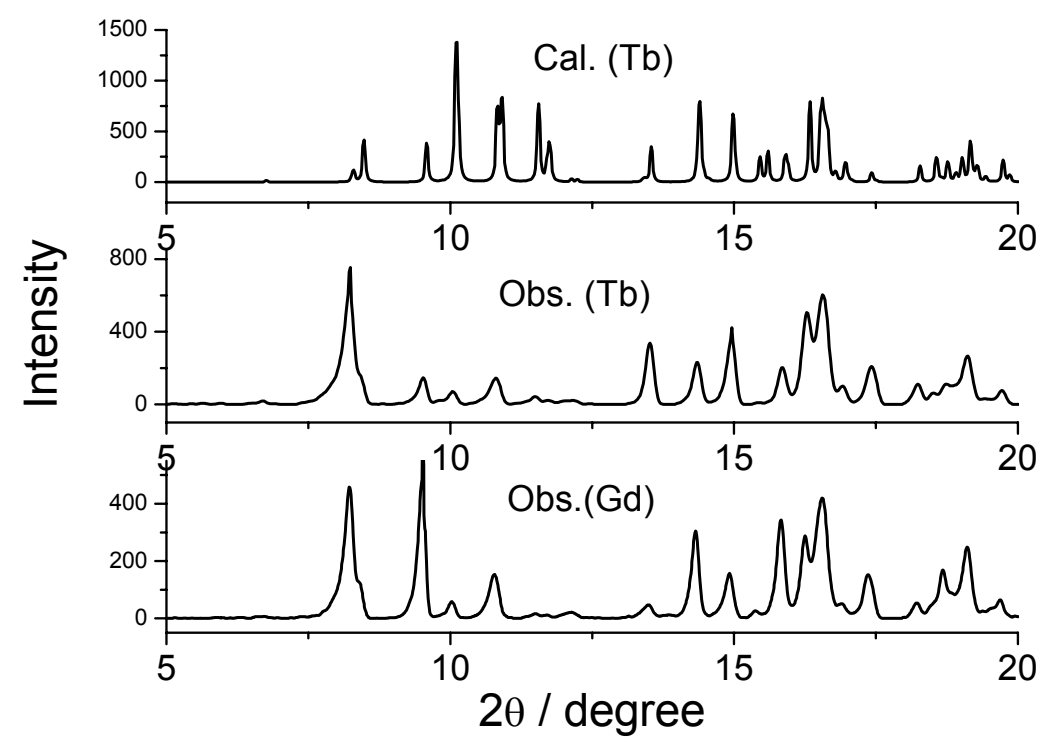

Figure S4. The calculated and observed powder XRD patterns of $\mathbf{1}$ and $\mathbf{4}$. 

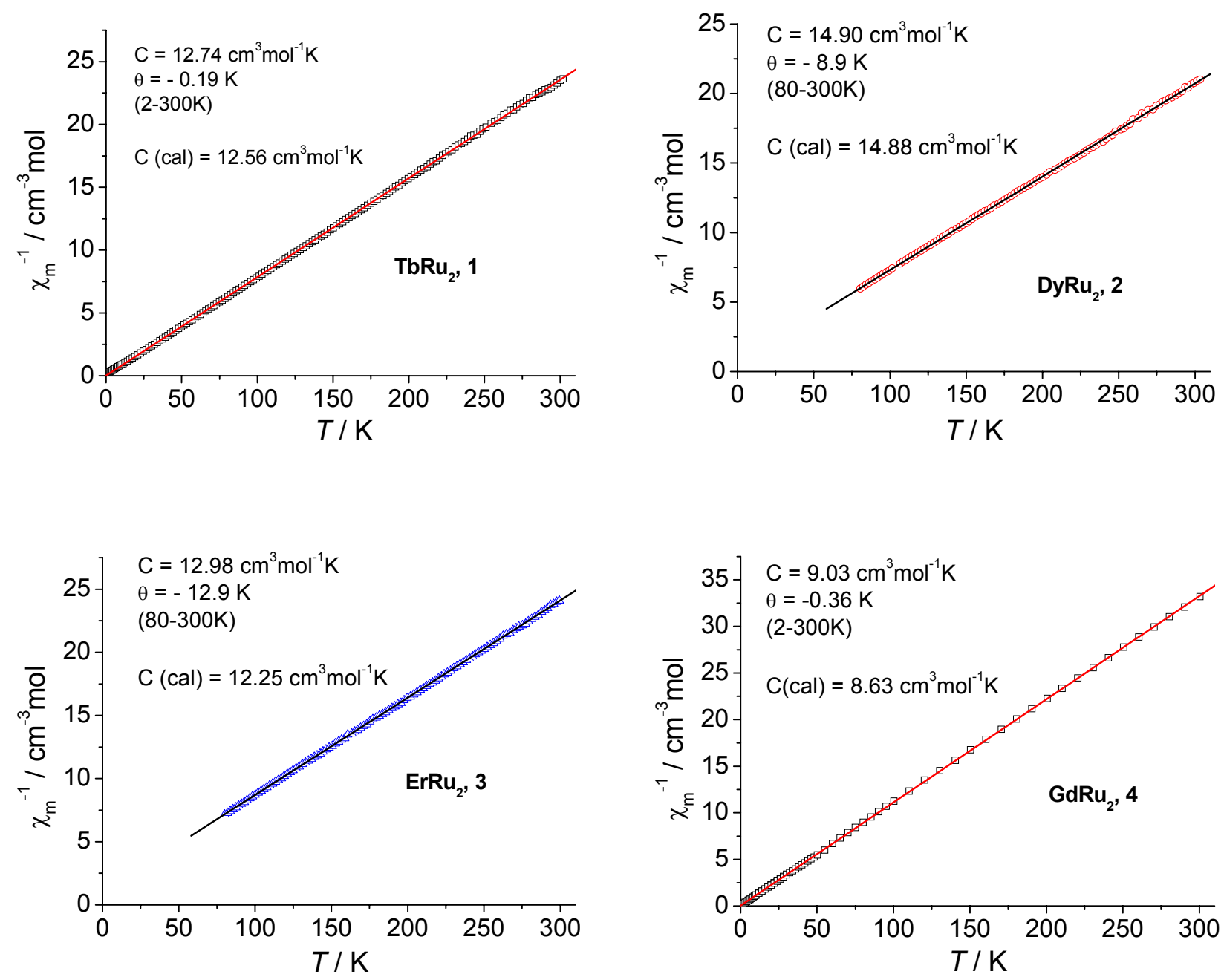

Figure S5. Temperature dependence of $\chi_{\mathrm{m}}^{-1}$ for compounds $\mathbf{1}-\mathbf{4}$. 